\title{
Gradient Controllability for Hyperbolic Systems
}

\author{
Hamid Bourray, Ali Boutoulout and Imad El Harraki* \\ Faculty of Sciences, Moulay Ismail University, Meknes, Morocco
}

Received: 18 Jul. 2013, Revised: 23 Sep. 2013, Accepted: 25 Sep. 2013

Published online: 1 Jan. 2014

\begin{abstract}
This paper deals with the problem of regional gradient controllability of hyperbolic systems. We show how one can reach a desired state gradient given only on a part of the system evolution domain. Also we explore a numerical approach using Hilbert Uniqueness Method (HUM) that leads to an explicit formula of the optimal control. The obtained results are successfully tested through computer simulations leading to some conjectures.
\end{abstract}

Keywords: hyperbolic systems, regional gradient controllability, strategic actuator, HUM approach.

\section{Introduction}

Many real systems are purely of distributed nature, and many of the systems conceived by humans are naturally very complex. Indeed, every complex problem always necessitates a complex solution. In spite of the fact that the most elaborated mathematical methods have been developed, a lot is to be done to bridge the gap between applied mathematics and the industrial world problems. The system theory contributed to fulfil this objective, and thus, obtain exploitable results in different domains. Particulary, the controllability is one of the most interesting notions of the system theory. Various previous researches treated the problem of controllability of hyperbolic systems which are composed of wave equation that we find in many real problems $[1,2]$. Copious works deal with the problem of steering a system (S) to a prescribed state defined on a space domain $\Omega$, were considered and studied in (Curtain and Zwart, 1995) [3], and the references therein. The study of controllability in hyperbolic systems was the subject of countless researches(Dolecki and Russell 1977, El Jai and Pritchard 1988, Lions 1988) ([4],[5]). The regional case was studied by Zerrik et al (2003) [6]. Pussed by the need to control the flux Zerrik et al.(1999)[7] developed the gradient controllability of parabolic systems. Our study will be devoted to the regional gradient controllability of the hyperbolic systems. This paper is organized as follows. Section 2 present a definition and characterization of regional controllability of hyperbolic systems. Section 3 defines the actuators gradient strategic and elaborates on its relationship with regional gradient controllability. Section 4 focusses on the approach devoted to the computation of the optimal control that permits to attain a gradient in a subregion $\omega$ of $\Omega$. At the last, the obtained results are successfully applied in one dimensional system with two numerical examples leading to some conjectures.

\section{Regional gradient controllability}

\subsection{Considered system}

Let $\Omega$ be an open bounded subset of $\mathbb{R}^{n}$ with regular boundary $\partial \Omega$. For $T>0$ we denote $Q=\Omega \times] 0, T[$, $\Sigma=\partial \Omega \times] 0, T[$ and we consider the hyperbolic system defined by

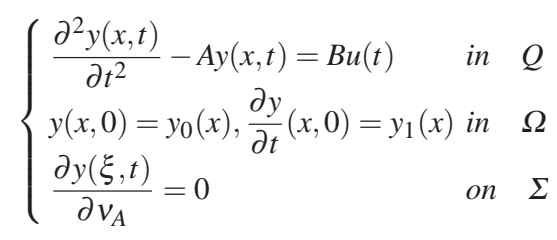

where $A$ is a second-order elliptic linear symmetric operator given by:

$$
\left\{\begin{array}{l}
A=-\sum_{i, j=1}^{n} \partial_{i}\left(a_{i j} \partial_{j}\right) \text { with } a_{i j}=a_{j i} \in \mathscr{C}^{1}(\Omega) \text { and there exists } \alpha>0 \\
\text { such that } \sum_{i, j=1}^{n} a_{i j} \xi_{i} \xi_{j} \geq \alpha \sum_{i=1}^{n}\left|\xi_{i}\right|^{2} \forall \xi=\left(\xi_{1}, \ldots, \xi_{n}\right) \in \mathbb{R}^{n}
\end{array}\right.
$$

\footnotetext{
*Corresponding author e-mail: imadharraki@gmail.com
} 
with domain

$$
D(A)=\left\{y \in H^{2}(\Omega), \frac{\partial y(\xi, t)}{\partial v_{A}}=0 \quad \text { on } \quad \partial \Omega\right\} .
$$

$B \in \mathscr{L}\left(U, H^{1}(\Omega)\right)$ where $U=L^{2}\left(0, T ; \mathbb{R}^{p}\right)$ and $p$ is the number of actuators, $\left(y_{0}, y_{1}\right) \in D(A) \times H^{1}(\Omega)$. We denote $\left(y_{u}(t), \frac{\partial y_{u}}{\partial t}(t)\right)$ the solution of the equation (1). If we denote by $\bar{A}=\left(\begin{array}{cc}0 & I \\ A & 0\end{array}\right)$ $z=\left(y, \frac{\partial y}{\partial t}\right)$ and $\bar{B} u=(0, B u)$ then the system $(1)$ can be written as:

$$
\left\{\begin{aligned}
\frac{\partial z(x, t)}{\partial t} & =\bar{A} z(x, t)+\bar{B} u(t) \text { in } \quad Q \\
z(0) & =\left(y_{0}, y_{1}\right)^{t} \text { in } \Omega
\end{aligned}\right.
$$

for all $\left(z_{1}, z_{2}\right) \in D(\bar{A})=D(A) \times H^{1}(\Omega)$ the solution of the system (4) is expressed using the semi-group $(\bar{S}(t))_{t \geq 0}$ generated by $\bar{A}$ and given by

$$
z(t)=\bar{S}(t) z_{0}+\int_{0}^{t} \bar{S}(t-\tau) \bar{B} u(\tau) d \tau
$$

With the assumption that the operator $A$ admits basis of eigenfunctions $w_{n_{j}}$ associated with the eigenvalues $\lambda_{n}$ of multiplicity $r_{n}$.

$$
\begin{aligned}
& \bar{S}(t) z(.)= \\
& \left(\begin{array}{l}
\sum_{n=1}^{\infty} \sum_{j=1}^{r_{n}}\left[\left\langle z_{1}, \omega_{n_{j}}\right\rangle \cos \left(\sqrt{-\lambda_{n}} t\right)+\frac{1}{\sqrt{-\lambda_{n}}}\left\langle z_{2}, \omega_{n_{j}}\right\rangle \sin \left(\sqrt{-\lambda_{n}} t\right)\right] \omega_{n_{j}}(.) \\
\sum_{n=1}^{\infty} \sum_{j=1}^{r_{n}}\left[\left(-\sqrt{-\lambda_{n}}\right)\left\langle z_{1}, \omega_{n_{j}}\right\rangle \sin \left(\sqrt{-\lambda_{n}} t\right)+\left\langle z_{2}, \omega_{n_{j}}\right\rangle \cos \left(\sqrt{-\lambda_{n}} t\right)\right] \omega_{n_{j}}(.)
\end{array}\right)
\end{aligned}
$$

and

$$
\left.\begin{array}{c}
\int_{0}^{t} \bar{S}(t-\tau) \bar{B} u(\tau) d \tau= \\
\left(\sum_{n=1}^{\infty} \sum_{j=1}^{r_{n}}\left[\int_{0}^{t} \frac{1}{\sqrt{-\lambda_{n}}}\left\langle B u(\tau), \omega_{n_{j}}\right\rangle \sin \left(\sqrt{-\lambda_{n}}(t-\tau)\right) d \tau\right] \omega_{n_{j}}(.)\right. \\
\sum_{n=1}^{\infty} \sum_{j=1}^{r_{n}}\left[\int_{0}^{t}\left\langle B u(\tau), \omega_{n_{j}}\right\rangle \cos \left(\sqrt{-\lambda_{n}}(t-\tau)\right) d \tau\right] \omega_{n_{j}}(.)
\end{array}\right)
$$

For $\omega \subset \Omega$ an open subregion of $\Omega$ with positive Lesbegue measure, let $\chi_{\omega}$ be the restriction function defined by

$$
\begin{aligned}
\chi_{\omega}:\left(L^{2}(\Omega)\right)^{n} \times\left(L^{2}(\Omega)\right)^{n} & \longrightarrow\left(L^{2}(\omega)\right)^{n} \times\left(L^{2}(\omega)\right)^{n} \\
\left(z_{1}, z_{2}\right) & \mapsto \chi_{\omega}\left(z_{1}, z_{2}\right)=\left.\left(z_{1}, z_{2}\right)\right|_{\omega}
\end{aligned}
$$

and $\chi_{\omega}^{*}$ denotes the adjoint operator, given by

$$
\begin{aligned}
\chi_{\omega}^{*}:\left(L^{2}(\omega)\right)^{n} \times\left(L^{2}(\omega)\right)^{n} & \longrightarrow\left(L^{2}(\Omega)\right)^{n} \times\left(L^{2}(\Omega)\right)^{n} \\
\left(z_{1}, z_{2}\right) & \mapsto \chi_{\omega}^{*}\left(z_{1}, z_{2}\right)= \begin{cases}\left(z_{1}, z_{2}\right) & \text { in } \omega \\
0 & \text { in } \Omega \backslash \omega\end{cases}
\end{aligned}
$$

Consider the operator $\nabla$ given by the formula

$$
\begin{aligned}
\nabla: H^{1}(\Omega) & \longrightarrow\left(L^{2}(\Omega)\right)^{n} \\
y & \mapsto \nabla y=\left(\frac{\partial y}{\partial x_{1}}, \ldots, \frac{\partial y}{\partial x_{n}}\right)
\end{aligned}
$$

And the operator $\tilde{\nabla}$ given by the formula

$$
\begin{aligned}
\tilde{\nabla}: H^{1}(\Omega) \times H^{1}(\Omega) & \longrightarrow\left(L^{2}(\Omega)\right)^{n} \times\left(L^{2}(\Omega)\right)^{n} \\
\left(y_{0}, y_{1}\right) & \mapsto \tilde{\nabla}\left(y_{0}, y_{1}\right)=\left(\nabla y_{0}, \nabla y_{1}\right)
\end{aligned}
$$

Let us give some definitions about the regional controllability of the gradient.

\subsection{Definition and properties}

\section{Definition 1.}

-The system (1) is said to be $\omega$-exactly gradient controllable if for all $\left(g_{p}^{d}, g_{s}^{d}\right) \in\left(L^{2}(\omega)\right)^{n} \times\left(L^{2}(\omega)\right)^{n}$ there exists $u \in U$ such that

$$
\chi_{\omega}\left(\nabla y_{u}(T), \nabla \frac{\partial y_{u}}{\partial t}(T)\right)=\left(g_{p}^{d}, g_{s}^{d}\right)
$$

-The system (1) is said to be w-weakly gradient controllable if for all $\varepsilon>0$ for all $\left(g_{p}^{d}, g_{s}^{d}\right) \in\left(L^{2}(\omega)\right)^{n} \times\left(L^{2}(\omega)\right)^{n}$ there exists $u \in U$ such that

$$
\left\|\chi_{\omega}\left(\nabla y_{u}(T), \nabla \frac{\partial y_{u}}{\partial t}(T)\right)-\left(g_{p}^{d}, g_{s}^{d}\right)\right\|_{\left(L^{2}(\omega)\right)^{n} \times\left(L^{2}(\omega)\right)^{n}} \leq \varepsilon
$$

Consider the operator

$$
\begin{aligned}
H: \quad L^{2}\left(0, T, \mathbb{R}^{p}\right) & \longrightarrow H^{1}(\Omega) \times H^{1}(\Omega) \\
u & \mapsto\left(y_{u}(T), \frac{\partial y_{u}}{\partial t}(T)\right)
\end{aligned}
$$

It is clear that the system (1) is $\omega$-exactly (resp. $\omega$-weakly) gradient controllable if

$$
\begin{gathered}
\operatorname{Im} \chi_{\omega} \tilde{\nabla} H=\left(L^{2}(\omega)\right)^{n} \times\left(L^{2}(\omega)\right)^{n} \\
\left(\text { resp. } \quad \operatorname{Im} \chi_{\omega} \tilde{\nabla} H=\left(L^{2}(\omega)\right)^{n} \times\left(L^{2}(\omega)\right)^{n} .\right)
\end{gathered}
$$

\section{Remark.}

1.Let $J(u)=\int_{0}^{T}\|u(t)\|_{\mathbb{R}^{p}}^{2} d t$ be the transfer cost . Then for any $\omega \subset \Omega$, the regional gradient transfer cost in $\omega$ is smaller than the transfer cost in $\Omega$.

2.The above definitions mean that we are only interested in the transfer of the system gradient to a desired function on the subregion $\omega \subset \Omega$.

3.If the system (1) is exactly gradient controllable in $\omega$ then it is weakly gradient controllable in $\omega$.

4.For $\omega_{2} \subset \omega_{1}$ the system (1) is exactly (resp. weakly) gradient controllable in $\omega_{1}$ then it is exactly (resp. weakly) gradient controllable in $\omega_{2}$.

\section{Proposition 1.}

1.The system (1) is $\omega$-exactly gradient controllable if and only if

$$
\operatorname{Ker} \chi_{\omega}+\operatorname{Im} \tilde{\nabla} H=\left(L^{2}(\Omega)\right)^{n} \times\left(L^{2}(\Omega)\right)^{n}
$$

2.The system (1) is $\omega$-weakly gradient controllable if and only if

$$
\operatorname{Ker} \chi_{\omega}+\overline{\operatorname{Im} \tilde{\nabla} H}=\left(L^{2}(\Omega)\right)^{n} \times\left(L^{2}(\Omega)\right)^{n}
$$


Proof.

1.Let $y \in\left(L^{2}(\Omega)\right)^{n} \times\left(L^{2}(\Omega)\right)^{n} \quad$ then $\chi_{\omega} y \in\left(L^{2}(\omega)\right)^{n} \times\left(L^{2}(\omega)\right)^{n}$ and since the system (1) is $\omega$ - exactly gradient controllable then there exist $u \in U$ such that $\chi_{\omega} y=\chi_{\omega} \tilde{\nabla} H u$. Let $y_{1}=y-\tilde{\nabla} H u$ and $y_{2}=\tilde{\nabla} H u$ then we have $y=y_{1}+y_{2}$ with $y_{1} \in \operatorname{Ker} \chi_{\omega}$ and $y_{2} \in \operatorname{Im} \tilde{\nabla} H$.

Conversely let $y \in\left(L^{2}(\omega)\right)^{n} \times\left(L^{2}(\omega)\right)^{n}$, then $\tilde{y}=\chi_{\omega}^{*} y \in\left(L^{2}(\Omega)\right)^{n} \times\left(L^{2}(\Omega)\right)^{n}$ which allows to write $\tilde{y}=y_{1}+y_{2}$ with $y_{1} \in \operatorname{Ker} \chi_{\omega}$ and $y_{2} \in \operatorname{Im} \tilde{\nabla} H$ consequently there exists $u \in U$ such that $y_{2}=\tilde{\nabla} H u$ therefore $\tilde{y}=y_{1}+\tilde{\nabla} H u$ which gives $y=\chi_{\omega} \tilde{\nabla} H u$ and thus the system (1) is $\omega$-exactly gradient controllable.

2.Let $y \in\left(L^{2}(\Omega)\right)^{n} \times\left(L^{2}(\Omega)\right)^{n} \quad$ then $\chi_{\omega} y \in\left(L^{2}(\omega)\right)^{n} \times\left(L^{2}(\omega)\right)^{n}$ and since the system (1) is $\omega$ - weakly gradient controllable then there exist $u_{n} \in U$ such that $\chi_{\omega} y=\lim \chi_{\omega} \tilde{\nabla} H u_{n}$ let $y_{1}=y-y_{2}$ with $y_{2}=\lim \tilde{\nabla} H u_{n}$ then we have $y=y_{1}+y_{2}$ with $y_{1} \in \operatorname{Ker} \chi_{\omega}$ and $y_{2} \in \overline{\operatorname{Im} \tilde{\nabla} H}$.

Conversely let $y \in\left(L^{2}(\omega)\right)^{n} \times\left(L^{2}(\omega)\right)^{n}$, then $\tilde{y}=\chi_{\omega}^{*} y \in\left(L^{2}(\Omega)\right)^{n} \times\left(L^{2}(\Omega)\right)^{n}$ which allows to write $\tilde{y}=y_{1}+y_{2}$ with $y_{1} \in \operatorname{Ker} \chi_{\omega}$ and $y_{2} \in \overline{\operatorname{Im} \tilde{\nabla} H}$ Consequently there exists $u_{n} \in U$ such that $y_{2}=\lim \tilde{\nabla} H u_{n}$ therefore

$\tilde{y}=y_{1}+\lim \tilde{\nabla} H u_{n}$ which gives $y=\lim \chi_{\omega} \tilde{\nabla} H u_{n}$ and thus the system (1) is $\omega$-weakly gradient controllable.

\section{Gradient controllability and actuators}

In this section we show that there exist a link between the regional gradient controllability and the actuators structure. Consider system (1) excited by $p$ zone actuators $\left(D_{i}, f_{i}\right)$ where $D_{i} \subset \Omega$ and $f_{i} \in H^{1}\left(D_{i}\right)$

$$
\begin{cases}\frac{\partial^{2} y(x, t)}{\partial t^{2}}-A y(x, t)=\sum_{i=1}^{p}\left(\chi_{D} f_{i}\right)(x) u_{i}(t) & \text { in } \quad Q \\ y(x, 0)=0, \frac{\partial y}{\partial t}(x, 0)=0 & \text { in } \Omega \\ \frac{\partial y(\xi, t)}{\partial v_{A}}=0 & \text { on } \Sigma\end{cases}
$$

\section{Definition 2.}

A sequence of the actuators is said to be gradient strategic if the excited system is weakly gradient controllable.

\section{Proposition 2.}

If the sequence of the actuators $\left(D_{i}, f_{i}\right)_{1 \leq i \leq p}$ is gradient strategic then

$$
\text { 1.p } \geq \sup \left(r_{m}\right) \text {. }
$$

$2 . \operatorname{Rank}\left(G_{m}\right)=r_{m}$, for all $m \geq 1$ where $\left(G_{m}\right)$ is the matrix of order $\left(p, r_{m}\right)$ given by

$$
\left(G_{m}\right)_{i, j}=\left\{\begin{array}{l}
\sum_{k=1}^{n}\left\langle\frac{\partial \omega_{m_{j}}}{\partial x_{k}}, f_{i}\right\rangle_{D_{i}} \text { zonal case } \\
\sum_{k=1}^{n} \frac{\partial \omega_{m_{j}}}{\partial x_{k}}\left(b_{i}\right) \quad \text { pointwise case }
\end{array}\right.
$$

Proof.

The proof will be developed in the internal zonal case.

The system (6) is weakly gradient controllable over $[0, T]$ then for all

$z=\left(z_{1}, z_{2}\right)$ in $\left(L^{2}(\Omega)\right)^{n} \times\left(L^{2}(\Omega)\right)^{n}$

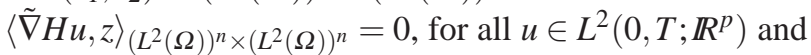
$T\rangle 0 \Longrightarrow z=0$. Consider the following system:

$$
\begin{cases}\frac{\partial^{2} \varphi(x, t)}{\partial t^{2}}-A^{*} \varphi(x, t)=0 & \text { in } Q \\ \varphi(x, T)=-h_{0} ; \varphi^{\prime}(x, T)=h_{0} & \text { in } \Omega \\ \varphi(\xi, t)=0 & \text { on } \Sigma\end{cases}
$$

Multiplying the system (7) by $\frac{\partial y}{\partial x_{k}}$ and integrating over $Q$ and using the green formula we obtain :

$$
-\left\langle\frac{\partial y^{\prime}}{\partial x_{k}}(T), \varphi(T)\right\rangle+\left\langle\frac{\partial y}{\partial x_{k}}(T), \varphi^{\prime}(T)\right\rangle=\sum_{i=1}^{p} \int_{0}^{T}\left\langle f_{i}, \frac{\partial \varphi}{\partial x_{k}}\right\rangle u_{i}(t) d t
$$$$
\text { then } \sum_{k=1}^{n}\left\langle\frac{\partial y^{\prime}}{\partial x_{k}}, h_{0}\right\rangle+\left\langle\frac{\partial y}{\partial x_{k}}, h_{0}\right\rangle=\sum_{k=1}^{n} \sum_{i=1}^{p} \int_{0}^{T}\left\langle f_{i}, \frac{\partial \varphi}{\partial x_{k}}\right\rangle u_{i}(t) d t
$$

$$
\langle\tilde{\nabla} H u,(I, I)\rangle=\sum_{k=1}^{n} \sum_{i=1}^{p} \int_{0}^{T}\left\langle f_{i}, \frac{\partial \varphi}{\partial x_{k}}\right\rangle u_{i}(t) d t
$$

with $I=\left(\begin{array}{c}h_{0} \\ \cdot \\ \cdot \\ \cdot \\ h_{0}\end{array}\right)$ and therefore

$$
\left[\begin{array}{cc}
\sum_{k=1}^{n} \sum_{i=1}^{p} \sum_{m}^{\infty} \sum_{j=1}^{r_{m}} \int_{0}^{T}\left(-\lambda_{m}\right)^{\frac{1}{2}}\left\langle-h_{0}, \omega_{m_{j}}\right\rangle\left\langle f_{i}, \frac{\partial \omega_{m_{j}}}{\partial x_{k}}\right\rangle \sin \left(\left(-\lambda_{n}\right)^{\frac{1}{2}}(\tau-T)\right) u_{i}(\tau) d \tau \\
\left.+\int_{0}^{T}\left\langle h_{0}, \omega_{m_{j}}\right\rangle\left\langle\frac{\partial \omega_{m_{j}}}{\partial x_{k}}, f_{i}\right\rangle \cos \left(\left(-\lambda_{n}\right)^{\frac{1}{2}}(\tau-T)\right) u_{i}(\tau) d \tau\right\} \\
=0 & \left.\forall u \in L^{2}\left(0, T ; \mathbb{R}^{p}\right) \text { and } T\right\rangle 0
\end{array}\right]
$$

$\Longrightarrow h_{0}=0$

this amounts to:

$$
\begin{aligned}
& {\left[\begin{array}{l}
\sum_{m}^{\infty}\left(-\lambda_{m}\right)^{\frac{1}{2}} \sin \left(-\lambda_{m}\right)^{\frac{1}{2}}(\tau-T) \sum_{j=1}^{r_{m}} \sum_{k=1}^{n}\left\langle-h_{0}, w_{m_{j}}\right\rangle\left\langle\frac{\partial \omega_{m_{j}}}{\partial x_{k}}, f_{i}\right\rangle \\
+\sum_{m}^{\infty} \cos \left(-\lambda_{m}\right)^{\frac{1}{2}}(\tau-T) \sum_{j=1}^{r_{m}} \sum_{k=1}^{n}\left\langle h_{0}, w_{m_{j}}\right\rangle\left\langle\frac{\partial \omega_{m_{j}}}{\partial x_{k}}, f_{i}\right\rangle=0 \\
1 \leq i \leq p
\end{array}\right]} \\
& \Longrightarrow h_{0}=0 \\
& \text { for } T \text { large enough },\{\cos (.-T), \sin (.-T)\} \quad \text { is an }
\end{aligned}
$$


orthonormal set of $L^{2}(0, T)$, then

$$
\left\{\begin{array}{l}
\left(-\lambda_{m}\right)^{\frac{1}{2}} \sum_{j=1}^{r_{n}} \sum_{k=1}^{n}\left\langle-h_{0}, \omega_{m_{j}}\right\rangle\left\langle\frac{\partial \omega_{m_{j}}}{\partial x_{k}}, f_{i}\right\rangle=0 \\
\text { and } \\
\sum_{j=1}^{r_{n}} \sum_{k=1}^{n}\left\langle h_{0}, \omega_{m_{j}}\right\rangle\left\langle\frac{\partial \omega_{m_{j}}}{\partial x_{k}}, f_{i}\right\rangle=0
\end{array}\right.
$$

So

$$
\sum_{j=1}^{r_{n}} \sum_{k=1}^{n}\left\langle h_{0}, \omega_{m_{j}}\right\rangle\left\langle\frac{\partial \omega_{m_{j}}}{\partial x_{k}}, f_{i}\right\rangle=0 \quad \Rightarrow h_{0}=0
$$

which concludes the proof.

\section{Regional target control}

The propose of this section is to explore an approach devoted to the computation of the optimal control of the system (6) to a given gradient in the subregion $\omega$. Suppose that $\left(g_{p}^{d}, g_{s}^{d}\right) \in \chi_{\omega}(\operatorname{Im}(\nabla) \times \operatorname{Im}(\nabla))$ is given and we set

$$
\bar{G}=\left\{\left(\phi_{0}, \phi_{1}\right) \in D(\Omega) \times D(\Omega) \mid \phi_{0}=\phi_{1}=0 \quad \text { sur } \quad \Omega \backslash \omega\right\}
$$

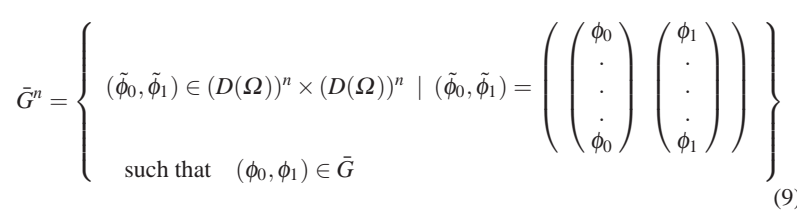

where $D(\Omega)$ is the space of test functions.

The problem is a follows: Does there exist a control $u \in U$ with minimum-norm such that for $\left(y_{0}, y_{1}\right) \in D(A) \times H^{1}(\Omega)$ and $f \in H^{1}(D)$

$$
\begin{cases}\frac{\partial^{2} y(x, t)}{\partial t^{2}}-\Delta y(x, t)=\left(\chi_{D} f\right)(x) u(t) & \text { in } \quad Q \\ y(x, 0)=y_{0}(x), \frac{\partial y}{\partial t}(x, 0)=y_{1}(x) & \text { in } \quad \Omega \\ \frac{\partial y(\xi, t)}{\partial v}=0 & \text { on } \quad \Sigma\end{cases}
$$

and the problem :

$$
\left\{\begin{array}{l}
\min _{u \in L^{2}(0, T)} J(u)=\|u\|_{L^{2}(0, T)}^{2} \\
\chi_{\omega}\left(\nabla y_{u}(T), \nabla \frac{\partial y_{u}}{\partial t}(T)\right)=\left(g_{p}^{d}, g_{s}^{d}\right)
\end{array}\right.
$$

where $\left(y_{u}\right)$ solution of (10)

\subsection{HUM approach}

The approach developed here is an extension of the Hilbert uniqueness method (HUM) developed by Lions (see[5])
For $\left(\phi_{1},-\phi_{0}\right) \in \bar{G}$, the system

$$
\begin{cases}\frac{\partial^{2} \phi(x, t)}{\partial t^{2}}-\Delta \phi(x, t)=0 & \text { in } Q \\ \phi(x, T)=\phi_{0}(x), \frac{\partial \phi}{\partial t}(x, T)=\phi_{1}(x) & \text { in } \Omega \\ \phi(\xi, t)=0 & \text { on } \quad \Sigma\end{cases}
$$

Has a unique solution (see[5]).

In $\bar{G}^{n}$ we define the following semi-norm :

$$
\left\|\left(\tilde{\phi}_{1},-\tilde{\phi}_{0}\right)\right\|_{\bar{G}^{n}}=\left(\int_{0}^{T}\left(\sum_{i=1}^{n}\left\langle\frac{\partial \phi(t)}{\partial x_{i}}, f\right\rangle_{L^{2}(D)}\right)^{2} d t\right)^{\frac{1}{2}}
$$

and we consider the system :

$\begin{cases}\frac{\partial^{2} \psi(x, t)}{\partial t^{2}}-\Delta \psi(x, t)=\sum_{i=1}^{n}\left\langle\frac{\partial \phi(t)}{\partial x_{i}}, f\right\rangle_{L^{2}(D)}\left(\chi_{D} f\right)(x) & \text { in } Q \\ \psi(x, 0)=y_{0}(x), \frac{\partial \psi}{\partial t}(x, 0)=y_{1}(x) & \text { in } \Omega \\ \frac{\partial \psi(\xi, t)}{\partial v}=0 & \text { on } \Sigma\end{cases}$

which has a unique solution such that $\left(\psi(T), \frac{\partial \psi}{\partial t}(T)\right) \in H^{2}(\Omega) \times H^{1}(\Omega)(\operatorname{see}[2])$

and

$\left(\psi(T), \frac{\partial \psi}{\partial t}(T)\right)=\left(\psi_{0}(T), \frac{\partial \psi_{0}}{\partial t}(T)\right)+\left(\psi_{1}(T), \frac{\partial \psi_{1}}{\partial t}(T)\right)$,

where $\psi_{0}$ and $\psi_{1}$ are solutions of the systems

$$
\begin{cases}\frac{\partial^{2} \psi_{0}(x, t)}{\partial t^{2}}-\Delta \psi_{0}(x, t)=0 & \text { in } \quad Q \\ \psi_{0}(x, 0)=y_{0}(x), \frac{\partial \psi_{0}}{\partial t}(x, 0)=y_{1}(x) & \text { in } \quad \Omega \\ \frac{\partial \psi_{0}(\xi, t)}{\partial v}=0 & \text { on } \quad \Sigma\end{cases}
$$

and

$$
\begin{cases}\frac{\partial^{2} \psi_{1}(x, t)}{\partial t^{2}}-\Delta \psi_{1}(x, t)=\sum_{i=1}^{n}\left\langle\frac{\partial \phi(t)}{\partial x_{i}}, f\right\rangle_{L^{2}(D)}\left(\chi_{D} f\right)(x) & \text { in } Q \\ \psi_{1}(x, 0)=0, \frac{\partial \psi_{1}}{\partial t}(x, 0)=0 & \text { in } \Omega \\ \frac{\partial \psi_{1}(\xi, t)}{\partial v}=0 & \text { on } \Sigma\end{cases}
$$

We consider the operator

$$
\Lambda\left(\tilde{\phi}_{1},-\tilde{\phi}_{0}\right)=\mathscr{P}\left(\nabla \psi_{1}(T), \nabla \psi_{1}^{\prime}(T)\right)
$$

is a symmetric and bounded operator where $\mathscr{P}=\chi_{\omega}^{*} \chi_{\omega}$. Then the regional gradient controllability problem turns up to solve the equation :

$$
\Lambda\left(\tilde{\phi}_{1},-\tilde{\phi}_{0}\right)=-\mathscr{P}\left(\nabla \psi_{0}(T), \nabla \psi_{0}^{\prime}(T)\right)+\chi_{\omega}^{*}\left(g_{p}^{d}, g_{s}^{d}\right)
$$

and we have the following result:

Theorem 1.If the system (10) is $\omega-$ weakly gradient controllable then (17)) has a unique solution $\left(\phi_{0}, \phi_{1}\right)$ and $u^{*}(t)=\sum_{i=1}^{n}\left\langle\frac{\partial \phi(t)}{\partial x_{i}}, f\right\rangle_{L^{2}(D)}$ drives the system (10) to 
$\left(g_{p}^{d}, g_{s}^{d}\right)$ on $\omega$ at time $T$, where $\phi$ is the solution of the system (12). Moreover, this control is the solution of the problem (11).

\section{Proof.}

Let $w_{i}(x)$ be the eigenfunctions of $\Delta$ associated with the eigenvalues $\lambda_{i}$. The mapping (13) defines a norm in $\bar{G}^{n}$. Indeed

$$
\left\|\left(\tilde{\phi}_{1},-\tilde{\phi}_{0}\right)\right\|_{\bar{G}^{n}}=0 \text { gives } \sum_{i=1}^{n}\left\langle\frac{\partial \phi}{\partial x_{i}}, f\right\rangle_{L^{2}(D)}^{2}=0 \text { on }[0, T]
$$

which is equivalent to

$\sum_{j=1}^{\infty}\left(\left\langle\phi_{0}, w_{j}\right\rangle \cos \left[\left(-\lambda_{i}\right)^{\frac{1}{2}}(t-T)\right]+\frac{\left\langle\phi_{1}, w_{j}\right\rangle}{\left(-\lambda_{j}\right)^{2}} \sin \left[\left(-\lambda_{j}\right)^{\frac{1}{2}}(t-T)\right]\right) \sum_{i=1}^{n}\left\langle\frac{\partial w_{j}}{\partial x_{i}}, f\right\rangle_{L^{2}(D)}=0$

thus, for $T$ large enough,$\{\cos (.-T), \sin (.-T)\}$ is an orthonormal set of $L^{2}(0, T)$, then (18) gives

$$
\left\langle\phi_{0}, w_{j}\right\rangle \sum_{i=1}^{n}\left\langle\frac{\partial w_{j}}{\partial x_{i}}, f\right\rangle_{L^{2}(D)}=0
$$

and

$$
\left\langle\phi_{1}, w_{j}\right\rangle \sum_{i=1}^{n}\left\langle\frac{\partial w_{j}}{\partial x_{i}}, f\right\rangle_{L^{2}(D)}=0
$$

since (the system (10) is $\omega$-weakly gradient controllable then $\sum_{i=1}^{n}\left\langle\frac{\partial w_{j}}{\partial x_{i}}, f\right\rangle_{L^{2}(D)} \neq 0$ and we have $\left\langle\phi_{0}, w_{j}\right\rangle=0$ and $\left\langle\phi_{1}, w_{j}\right\rangle=0, \forall i \geq 1$. It follows that $\phi_{0}=\phi_{1}=0$ and (13) is a norm. Let $\widehat{G}$ be the completion of $\bar{G}^{n}$ by the norm (13) and $\widehat{G}^{*}$ its dual. We show that $\Lambda$ is an isomorphism from $\widehat{G}$ into $\widehat{G}^{*}$. Indeed

$\left\langle\Lambda\left(\tilde{\phi}_{1},-\tilde{\phi}_{0}\right),\left(\tilde{\phi}_{1},-\tilde{\phi}_{0}\right)\right\rangle=\sum_{i=1}^{n}\left(\left\langle\frac{\partial \psi_{1}}{\partial x_{i}}(T), \phi_{1}\right\rangle-\left\langle\frac{\partial \psi_{1}^{\prime}}{\partial x_{i}}(T), \phi_{0}\right\rangle\right)$ multiplying (12) by $\frac{\partial \psi_{1}}{\partial x_{i}}$ and using of the Green formula we have.

$$
\int_{0}^{T}\left\langle\frac{\partial \phi(t)}{\partial x_{i}}, f\right\rangle_{L^{2}(D)} \sum_{j=1}^{n}\left\langle\frac{\partial \phi}{\partial x_{j}}, f\right\rangle_{L^{2}(D)}^{2} d t=\left\langle\frac{\partial \psi_{1}}{\partial x_{i}}(T), \phi_{1}\right\rangle-\left\langle\frac{\partial \psi_{1}^{\prime}}{\partial x_{i}}(T), \phi_{0}\right\rangle
$$

So we have $\left\langle\Lambda\left(\tilde{\phi}_{1},-\tilde{\phi}_{0}\right),\left(\tilde{\phi}_{1},-\tilde{\phi}_{0}\right)\right\rangle=\left\|\left(\tilde{\phi}_{1},-\tilde{\phi}_{0}\right)\right\|_{\widehat{G}}^{2}$. Hence, (17) has only one solution $\left(\tilde{\phi_{1}}, \tilde{\phi_{0}}\right)$ and $\sum_{i=1}^{n}\left\langle\frac{\partial \phi}{\partial x_{i}}, f\right\rangle_{L^{2}(D)}^{2}$ steers the system (10) to the desired gradient $\left(g_{p}^{d}, g_{s}^{d}\right)$ on $\omega$ at time $T$. Now we consider $U_{a d}=\left\{u \in U \mid \chi_{\omega}\left(\nabla y_{u}(T), \nabla \frac{\partial y_{u}}{\partial t}(T)\right)=\left(g_{p}^{d}, g_{s}^{d}\right)\right\}$.

For $v \in U_{a d}$ and under (11) we have

$$
\begin{aligned}
J^{\prime}\left(u^{*}\right)\left(v-u^{*}\right) & =2 \int_{0}^{T} u^{*}(t)\left(v(t)-u^{*}(t) d t\right. \\
& =2 \int_{0}^{T} \sum_{i=1}^{n}\left\langle\frac{\partial \phi}{\partial x_{i}}, f\right\rangle_{L^{2}(D)}^{2}\left(v(t)-u^{*}(t)\right) d t
\end{aligned}
$$

Applying Green's formula after multiplying (12) by $\frac{\partial\left(y_{u}-y_{v}\right)}{\partial x_{i}}$, and from the boundary and initial conditions we have :

$$
\begin{aligned}
\int_{0}^{T}\left\langle\frac{\partial \phi(t)}{\partial x_{i}}, f\right\rangle_{L^{2}(D)}^{2}\left(v(t)-u^{*}(t)\right) d t & =\left\langle\frac{\partial y_{u}}{\partial t}(T)-\frac{\partial y_{v}}{\partial t}(T), \frac{\partial \phi}{\partial x_{i}}(T)\right\rangle \\
& -\left\langle\frac{\partial y_{u}}{\partial t}(0)-\frac{\partial y_{v}}{\partial t}(0), \frac{\partial \phi}{\partial x_{i}}(0)\right\rangle \\
& +\left\langle y_{u}(0)-y_{v}(0), \frac{\partial \phi^{\prime}}{\partial x_{i}}(0)\right\rangle \\
& -\left\langle y_{u}(T)-y_{v}(T), \frac{\partial \phi^{\prime}}{\partial x_{i}}(T)\right\rangle
\end{aligned}
$$

so $\int_{0}^{T} \sum_{i=1}^{n}\left\langle\frac{\partial \phi(t)}{\partial x_{i}}, f\right\rangle_{L^{2}(D)}^{2}\left(v(t)-u^{*}(t)\right) d t=0$ Hence, $J^{\prime}\left(u^{*}\right)\left(v-u^{*}\right)=0$.

The uniqueness of $u^{*}$ comes from the strict convexity of $J$ and establishes its optimality.

\subsection{Numerical approach}

In this section we give an approach which gives explicit formulae for $\tilde{\phi}_{0}, \hat{\phi}_{1}$ and the optimal control solution of (11). We have seen that the problem (11) can be used to solve (17), which is equivalent to solving the minimization problem

$$
\inf _{\left(\tilde{\phi}_{1}, \tilde{\phi_{0}}\right) \in \widehat{G}} R\left(\tilde{\phi_{1}}, \tilde{\phi_{0}}\right)
$$

where $R$ is given by

$$
\begin{aligned}
R\left(\tilde{\phi_{1}}, \tilde{\phi_{0}}\right) & =\frac{1}{2} \int_{0}^{T}\left(\sum_{i=1}^{n}\left\langle\frac{\partial \phi}{\partial x_{i}}, f\right\rangle_{L^{2}(D)}\right)^{2} d t+\sum_{i=1}^{n}\left\langle\frac{\partial \psi_{0}}{\partial x_{i}}(T), \phi_{1}\right\rangle \\
& -\left\langle\frac{\partial \psi_{0}^{\prime}}{\partial x_{i}}(T), \phi_{0}\right\rangle-\left\langle g_{p_{i}}^{d}, \phi_{1}\right\rangle+\left\langle g_{s_{i}}^{d}, \phi_{0}\right\rangle
\end{aligned}
$$

Expanding the integrand and letting $T \rightarrow+\infty$, we obtain:

$\frac{1}{2 T} \int_{0}^{T}\left(\sum_{i=1}^{n}\left\langle\frac{\partial \phi}{\partial x_{i}}, f\right\rangle_{L^{2}(D)}\right)^{2} d t=\sum_{j=1}^{\infty} \frac{1}{4}\left(\left\langle\phi_{0}, w_{j}\right\rangle^{2}-\frac{1}{\lambda_{j}}\left\langle\phi_{0}, w_{j}\right\rangle^{2}\right)\left(\sum_{i=1}^{n}\left\langle f, \frac{\partial w_{j}}{\partial x_{i}}\right\rangle_{L^{2}(D)}\right)^{2}$

Thus, for $T$ large enough, we obtain :

$\frac{1}{2} \int_{0}^{T}\left(\sum_{i=1}^{n}\left\langle\frac{\partial \phi}{\partial x_{i}}, f\right\rangle_{L^{2}(D)}\right)^{2} d t \simeq \sum_{j=1}^{\infty} \frac{T}{4}\left(\left\langle\phi_{0}, w_{j}\right\rangle^{2}-\frac{1}{\lambda_{j}}\left\langle\phi_{1}, w_{j}\right\rangle^{2}\right)\left(\sum_{i=1}^{n}\left\langle f, \frac{\partial w_{j}}{\partial x_{i}}\right\rangle_{L^{2}(D)}\right)^{2}$

The problem (19) can allow us to minimize the functional $R$ given by :

$$
\begin{aligned}
R\left(\phi_{0}, \phi_{1}\right) & \simeq \sum_{j=1}^{\infty} \frac{T}{4}\left\langle\phi_{0}, w_{j}\right\rangle^{2}\left(\sum_{i=1}^{n}\left\langle f, \frac{\partial w_{j}}{\partial x_{i}}\right\rangle_{L^{2}(D)}\right)^{2}-\sum_{i=1}^{n}\left\langle\frac{\partial \psi_{0}^{\prime}}{\partial x_{i}}(T), w_{j}\right\rangle\left\langle\phi_{0}, w_{j}\right\rangle \\
& +\left\langle\phi_{0}, w_{j}\right\rangle\left\langle g_{s_{i}}^{d}, w_{j}\right\rangle \\
& +\sum_{j=1}^{\infty} \frac{-T}{4 \lambda_{j}}\left\langle\phi_{1}, w_{j}\right\rangle^{2}\left(\sum_{i=1}^{n}\left\langle f, \frac{\partial w_{j}}{\partial x_{i}}\right\rangle_{L^{2}(D)}\right)^{2}+\left\langle\frac{\partial \psi_{0}}{\partial x_{i}}(T), w_{j}\right\rangle\left\langle\phi_{1}, w_{j}\right\rangle \\
& -\left\langle g_{p_{i}}^{d}, w_{j}\right\rangle\left\langle\phi_{1}, w_{j}\right\rangle
\end{aligned}
$$

The first term of (22) is independent of $\left\langle\frac{\partial \phi}{\partial t}(0), w_{j}\right\rangle$ and the second term is independent de $\left\langle\phi(0), w_{j}\right\rangle$. Hence we can minimize

$\frac{T}{4}\left\langle\phi_{0}, w_{j}\right\rangle_{\omega}^{2}\left(\sum_{i=1}^{n}\left\langle f, \frac{\partial w_{j}}{\partial x_{i}}\right\rangle_{L^{2}(D)}\right)^{2}-\sum_{i=1}^{n}\left\langle\frac{\partial \psi_{0}^{\prime}}{\partial x_{i}}(T), w_{j}\right\rangle\left\langle\phi_{0}, w_{j}\right\rangle_{\omega}+\left\langle\phi_{0}, w_{j}\right\rangle_{\omega}\left\langle g_{s_{i}}^{d}, w_{j}\right\rangle$ 
and

$-\frac{T}{4 \lambda_{j}}\left\langle\phi_{1}, w_{j}\right\rangle_{\omega}^{2}\left(\sum_{i=1}^{n}\left\langle f, \frac{\partial w_{j}}{\partial x_{i}}\right\rangle_{L^{2}(D)}\right)^{2}+\sum_{i=1}^{n}\left\langle\frac{\partial \psi_{0}}{\partial x_{i}}(T), w_{j}\right\rangle\left\langle\phi_{1}, w_{j}\right\rangle_{\omega}-\left\langle g_{p_{i}}^{d} w_{j}\right\rangle\left\langle\phi_{1}, w_{j}\right\rangle_{\omega}$

which gives

$$
\left\{\begin{array}{c}
\left\langle\phi_{0}, w_{j}\right\rangle_{\omega}=\frac{2}{T} \frac{\sum_{i=1}^{n}\left(\left\langle\frac{\partial \psi_{0}^{\prime}}{\partial x_{i}}(T), w_{j}\right\rangle-\left\langle g_{s_{i}}^{d}, w_{j}\right\rangle\right)}{\left(\sum_{i=1}^{n}\left\langle f, \frac{\partial w_{j}}{\partial x_{i}}\right\rangle_{L^{2}(D)}\right)^{2}} \\
\left\langle\phi_{1}, w_{j}\right\rangle_{\omega}=\frac{2 \lambda_{j}}{T} \frac{\sum_{i=1}^{n}\left(\left\langle\frac{\partial \psi_{0}}{\partial x_{i}}(T), w_{j}\right\rangle-\left\langle g_{p_{i}}^{d}, w_{j}\right\rangle\right)}{\left(\sum_{i=1}^{n}\left\langle f, \frac{\partial w_{j}}{\partial x_{i}}\right\rangle_{L^{2}(D)}\right)^{2}}
\end{array}\right.
$$

Then we obtain

$$
\phi_{0}= \begin{cases}\frac{2}{T} \sum_{j=1}^{\infty} \frac{\sum_{i=1}^{n}\left(\left\langle\frac{\partial \psi_{0}^{\prime}}{\partial x_{i}}(T), w_{j}\right\rangle-\left\langle s_{s_{i}}^{d}, w_{j}\right\rangle\right)}{\left(\sum_{i=1}^{n}\left\langle f, \frac{\partial w_{j}}{\partial x_{i}} L_{L^{2}(D)}\right)^{2}\right.} w_{j}(x) & x \in \omega \\ 0 & x \in \Omega \backslash \omega\end{cases}
$$

and

$$
\phi_{1}= \begin{cases}\frac{2}{T} \sum_{j=1}^{\infty} \lambda_{j} \frac{\sum_{i=1}^{n}\left(\left\langle\frac{\partial \psi_{0}^{\prime}}{\partial x_{i}}(T), w_{j}\right\rangle-\left\langle g_{p_{i}}^{d}, w_{j}\right\rangle\right)}{\left(\sum_{i=1}^{n}\left\langle f, \frac{\partial w_{j}}{\partial x_{i}}\right\rangle_{L^{2}(D)}\right)^{2}} w_{j}(x) & x \in \omega \\ 0 & x \in \Omega \backslash \omega\end{cases}
$$

and the optimal control which steers the system (11) to the desired gradient $\left(g_{p}^{d}, g_{s}^{d}\right)$ in $\omega$ at time $T$ is given by

$u^{*}(t)=\sum_{i=1}^{+\infty}\left[\left\langle\phi_{0}, w_{i}\right\rangle \cos \left(\sqrt{-\lambda_{i}}(t-T)\right)+\frac{\left\langle\phi_{1}, w_{i}\right\rangle}{\sqrt{-\lambda_{i}}} \sin \left(\sqrt{-\lambda_{i}}(t-T)\right)\right]\left\langle\sum_{l=1}^{n} \frac{\partial w_{i}}{\partial x_{l}}, f\right\rangle_{L^{2}(D)}$

We define a final error (depending on the subregion $\omega$ and the location of the actuator) by considering $\mathscr{E}=\left\|\nabla y_{u}(T)-g_{p}^{d}\right\|_{\left(L^{2}(\omega)\right)^{n}}^{2}+\left\|\nabla y_{u}^{\prime}(T)-g_{s}^{d}\right\|_{\left(L^{2}(\omega)\right)^{n}}^{2}$.

$\phi_{0}, \phi_{1}$ and $u^{*}$ are given by (24), (25) and (26). The general algorithm for computing the optimal control for (10) is as follows.

\section{Algorithm}

1.Choose actuator location $D \subset \Omega$, the subregion $\omega$ and precision $\varepsilon$.

2.Choose approximation order $M$.

3.Calculation of $\phi_{0}$ and $\phi_{1}$ using (24), (25) and $u^{*}$ from (26).

4.Solve (12) and obtaining $\nabla y_{u}(T)$ and $\nabla y_{u}^{\prime}(T)$.

5.If $\mathscr{E} \leq \varepsilon$ stop, else $M \leftarrow M+1$ and return to step 3 .

\section{Simulation results}

Here we consider one-dimensional system excited by one internal pointwise actuator

$$
\left\{\begin{array}{lll}
\frac{\partial^{2} y(x, t)}{\partial t^{2}}-\frac{\partial^{2} y(x, t)}{\partial x^{2}}=\delta(x-b) u(t) & \text { in } & ] 0,1[\times] 0, T[ \\
y(x, 0)=y_{0}(x), \quad \frac{\partial y}{\partial t}(x, 0)=y_{1}(x) & \text { in }] 0,1[ \\
y(0, t)=y(1, t)=0 & \text { on }] 0, T[.
\end{array}\right.
$$

Because of linearity of the above system, we take $y_{0}(x)=y_{1}(x)=0$.

For $T=2$ and $\mathrm{b}=0.23$, we have the following results:

\subsection{Example1}

Here we test the previous algorithm with the desired gradient position and speed gradient given by

$$
\left\{\begin{array}{l}
g_{p}^{d}(x)=2 \sin (\pi x) /\left(x^{4}+1\right) \\
g_{s}^{d}(x)=8 \sin (\pi x) x^{2}
\end{array}\right.
$$

\section{Global target}

For $\omega=] 0,1[$ we have the figures:

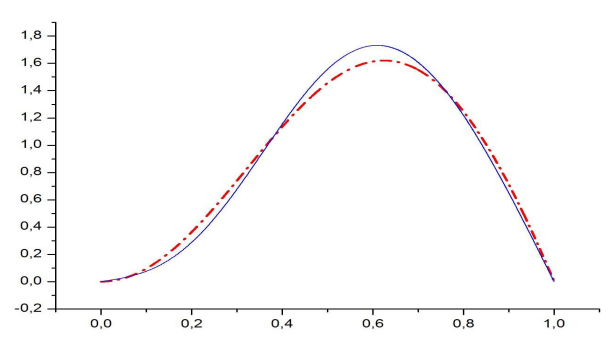

Figure 1: The desired position gradient $g_{p}^{d}$ (dashed line) and its reached (solid line) in $\omega$.

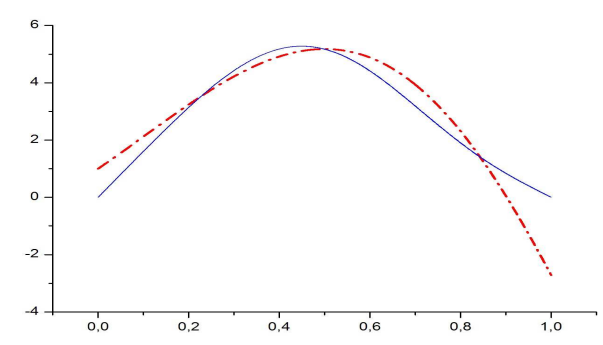

Figure 2: The desired speed gradient $g_{s}^{d}$ (dashed line) and its reached (solid line) in $\omega$.

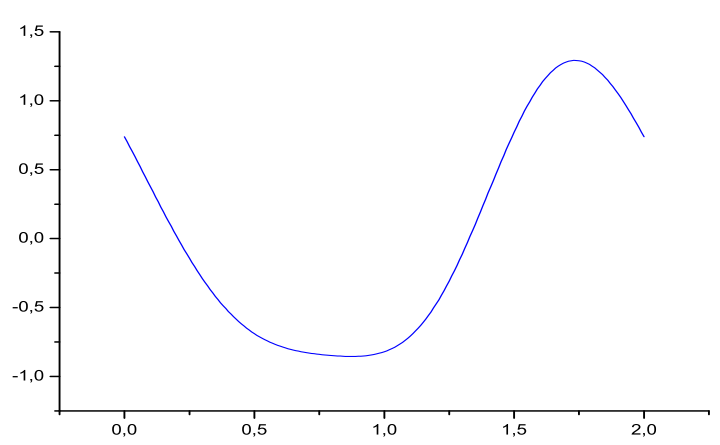

Figure 3: The evolution of the control function. 


\section{Regional target}

For $\omega=] 0.3,0.5$, we have the following figures

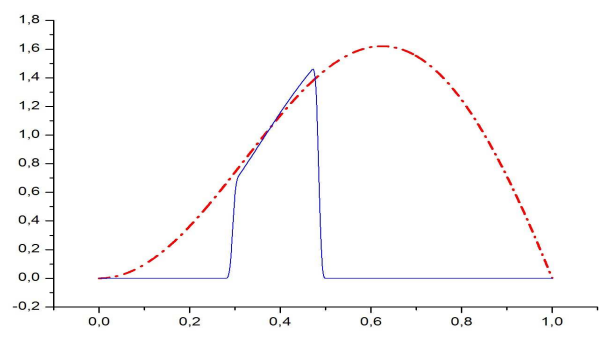

Figure 4: The desired position gradient $g_{p}^{d}$ (dashed line) and its reached (solid line) in $\omega$.

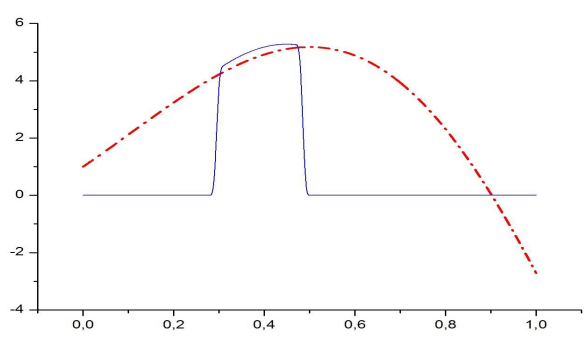

Figure 5: The desired speed gradient $g_{s}^{d}$ (dashed line) and its reached (solid line) in $\omega$

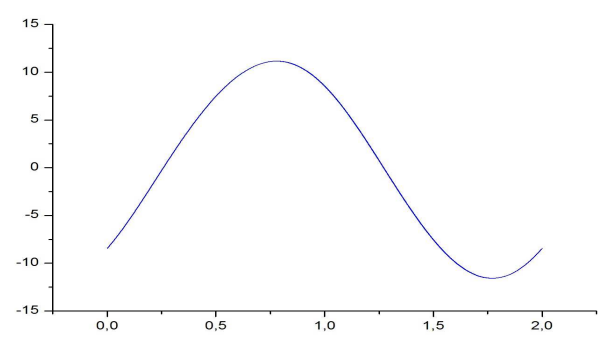

Figure 6: The evolution of the control function.

Fig 4 and Fig 5 show that the reached position gradient (resp. speed gradient) is very close to the desired gradient position (resp. gradient speed) in $\omega$. The reached state gradient and speed gradient are obtained with the error $\varepsilon=2.7631 \times 10^{-3}$ and the cost $J\left(u^{*}\right)=2.32 \times 10^{-1}$.

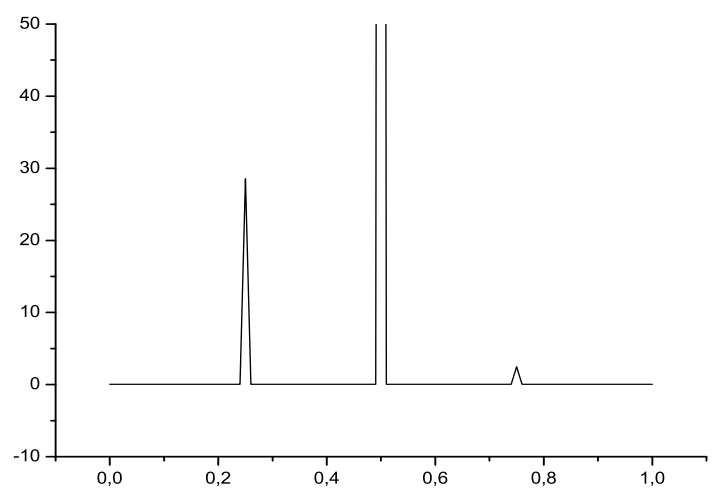

Figure 7: The reconstruction error with respect to the actuator location.

The following simulation results show the evolution of the reconstruction error with respect to the actuator location $b$ in $] 0,1[$.

Figure 7 reveals the following facts:

-For a given subregion $\omega$, there is an optimal actuator location (optimal in the sense that it leads to a desired state gradient very close to the reached one).

-When an actuator is located sufficiently far from the subregion $\omega$, the reconstructed gradient error is constant for any locations

Relation between the subregion area and reconstruction error

Here we study the evolution of the reconstruction error with respect to the subregion area.

Table 1: Evolution of the error with respect to the subregion area.

\begin{tabular}{|c|c|}
\hline subregion & Reconstruction error \\
\hline \hline$] 0.1,0.9[$ & $4.0005 \times 10^{-3}$ \\
] $0.1,0.8[$ & $3.7310 \times 10^{-3}$ \\
] $0.1,0.7[$ & $3.6562 \times 10^{-3}$ \\
] $0.2,0.7[$ & $3.3631 \times 10^{-3}$ \\
] $0.3,0.6[$ & $2.7631 \times 10^{-3}$ \\
] $0.3,0.5[$ & $1.2693 \times 10^{-4}$ \\
]0.3,0.4[ & $1.0971 \times 10^{-5}$ \\
\hline
\end{tabular}

We note that the reconstruction error depends on the area of the subregion. Its means that the greater the area is the greater the error is.

\subsection{Example2}

Here the considered position and speed gradient are given by

$$
\left\{\begin{array}{l}
g_{p}^{d}(x)=\pi \sin (\pi x) \tan (x) \\
g_{s}^{d}(x)=(\pi \sin (\pi x)+\cos (\pi x)) \exp (x)
\end{array}\right.
$$

Global target

For $\omega=] 0,1[$ we have the figures: 


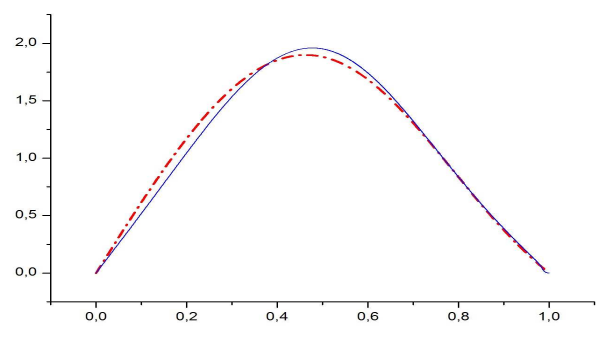

Figure 8: The desired position gradient $g_{p}^{d}$ (dashed line) and its reached (solid line) in $\omega$.

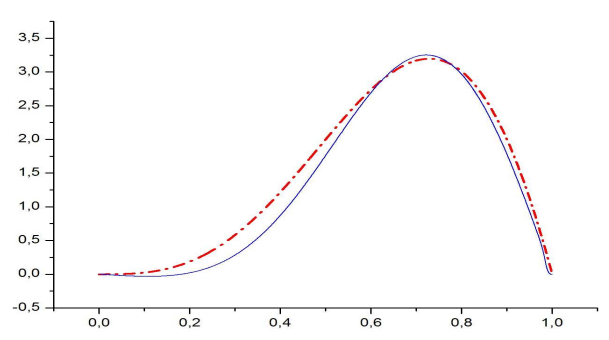

Figure 9: The desired speed gradient $g_{s}^{d}$ (dashed line) and its reached (solid line) in $\omega$.

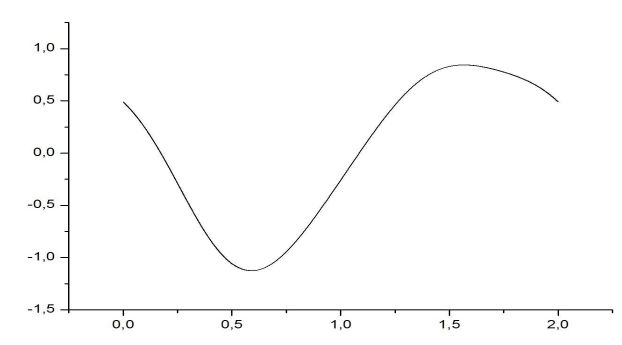

Figure 10: The evolution of the control function.

\section{Regional target}

For $\omega=] 0.65,1[$, we have the following figures

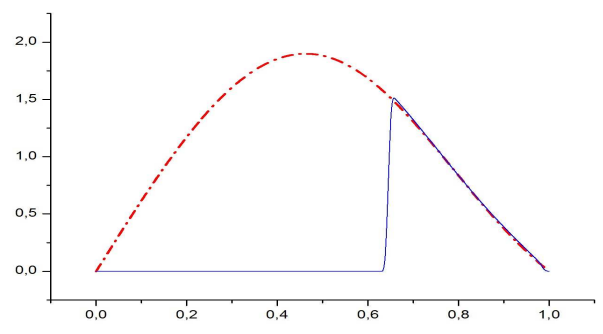

Figure 11: The desired position gradient $g_{p}^{d}$ (dashed line ) and its reached (solid line) in $\omega$.

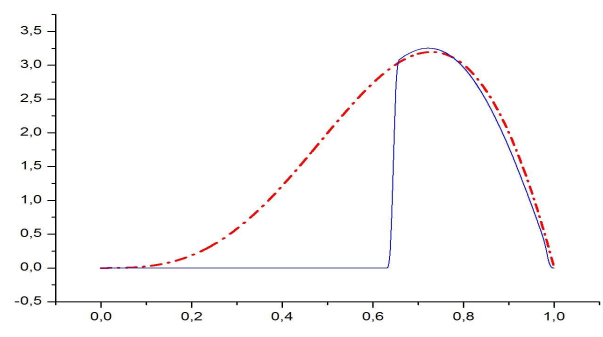

Figure 12: The desired speed gradient $\frac{\partial y^{\prime}(T)}{\partial x}$ (dashed line ) and its reached (solid line ) in $\omega$.

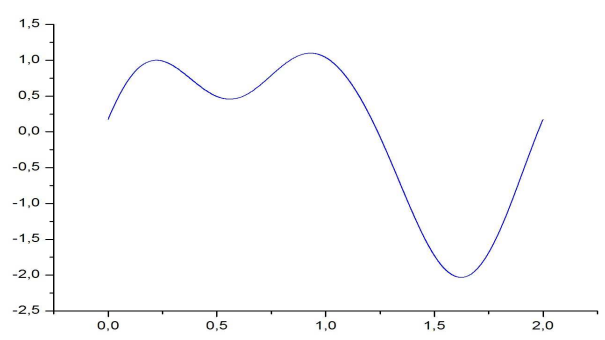

Figure 13: The evolution of the control function.

Figures 11 and 12 show that the reached position gradient (resp. speed gradient) is very close to the desired gradient position (resp. gradient speed) in $\omega$.

The reached state and speed gradient are obtained with the error $\varepsilon=5.1419 \times 10^{-3}$ and the cost $J\left(u^{*}\right)=3.17 \times 10^{-1}$

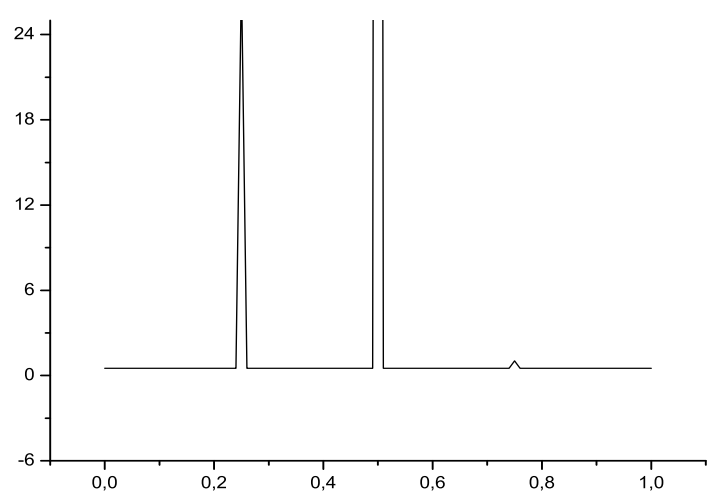

Figure 14: The reconstruction error with respect to the actuator location.

In this example, we examined the evolution of the reconstruction error, with respect to the actuator location, we obtained similar conclusion as in the example 1.

\section{Conclusion}

In this work we have extended the notion of regional gradient controllability to hyperbolic systems. We gave 
definitions and important characterizations in connection with strategic actuator and which allowed as to extend the HUM approach and then achieve the desired gradient. A minimization problem is also considered which provided us an algorithm with explicit formula of the optimal control that is performed through numerical examples and simulations. The problem where the subregion target is a part of the boundary of the system evolution domain, is of great interest and the work is under consideration and will be the subject of the feature paper. We are also interested to control the gradient of semilinear systems which are very close to nonlinear ones, and then we try to extend the existed results given in observability (see[8]and[9]) and controllability (see[10]) of semilinear systems to gradient case.

\section{References}

[1] E. Zerrik, H. Bourray and S. Ben Hadid, 2010 Sensors and boundary state reconstruction of hyperbolic systems, Int. J. Appl. Math. Comput. Sci., 20, 227-238 (2010).

[2] J. L. Lions, E. Magenes, , Problèmes aux limites non homogénes et applications., 1, (1968).

[3] R. F. Curtain and H. Zwart, An introduction to infinite dimentional linear systems theory, Springer Verlag, New York, (1995).

[4] A. El Jai and A.J Prichard, Sensors and actuators in distributed systems analysis, Ellis Horwood series in applied mathematics, J. Wiley., (1988).

[5] J. L. Lions, Contrôlabilité éxacte perturbation et stabilisation des systèmes distribués non homognes et applications. Tome 1, Masson, (1988).

[6] E. Zerrik and R. Larhrissi, Regional target control of the wave equation, Int. J. Systems Science, 32, 1233-1242 (2001).

[7] E. Zerrik and A. Boutoulout and A. Kamal, Regional gradient controllability of parabolic systems, Int. J. appl. Math and Comp. Sci, 9, 767-787 (1999).

[8] A. Boutoulout, H. Bourray and F. El Alaoui, Boundary gradient observability for semilinear parabolic systems: Sectorial approach, Math. Sci. Lett., 2, 45-53 (2013).

[9] A. Boutoulout, H. Bourray and F. El Alaoui, Boundary flux reconstruction for semilinear parabolic systems: HUM Approach, International Review of Automatic Control (I.RE.A.CO.,) 5, 652-659 (2012).

[10] A. Kamal, A. Boutoulout and S. A Ould Beinane, Regianl boundary controllabiity of semilinear distributed parabolic systems, International Review of Automatic Control(I.RE.A.CO.,) 3, 378-387 (2010).

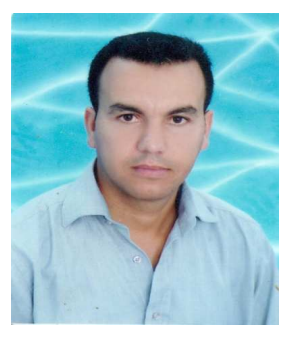

Hamid Bourray is a professor at the University Moulay Ismail of Meknes in Morocco. $\mathrm{He}$ is an assistant professor at the same University. He got his Doctorat in Systems Analysis (2002) at the Faculty of Sciences in Meknes. Professor Bourray has published many paper in the area of system analysis and control. He is a researcher at STI Team, Macs Laboratory at the University Moulay Ismail of Meknes in Morocco.

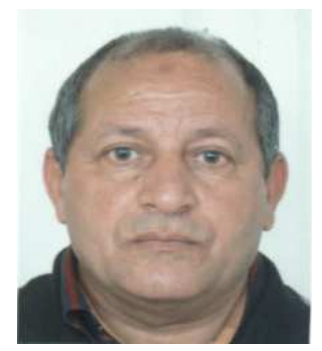

Ali Boutoulout is a professor at the University Moulay Ismail of Meknes in Morocco. He obtained his Doctorat d'Etat in System Regional Analysis (2000) at University Moulay Ismail. Professor Boutoulout has published many paper in the area of system analysis and control. Now he is the head of the research team STI (System Theory and Informatics) and a director of Master System Theory and Informatics, in department of Mathematics and Informatics of Faculty of Sciences at the University Moulay Ismail of Meknes in Morocco.

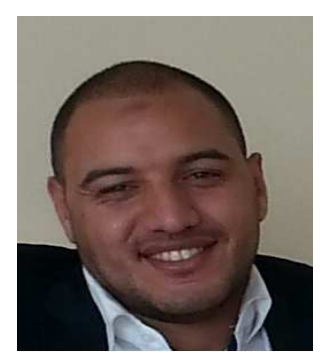

Imad Harraki is
researcher, preparing his Doctorat in Applied Mathematics at the University Moulay Ismail of Meknes in Morocco. His research area is focused on analysis and control of distributed systems and numerical analysis. At present he is a searcher at STI Team, Macs Laboratory at University Moulay Iamail of Meknes, Morocco. 